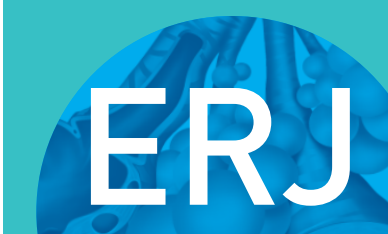

open research

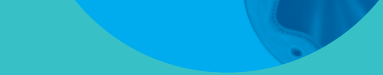

\title{
Is idiopathic pulmonary fibrosis a cancer-like disease? Transcriptome analysis to fuel the debate
}

\section{To the Editor:}

Idiopathic pulmonary fibrosis (IPF) is a devastating and eventually fatal diffuse parenchymal lung disorder that largely remains refractory to pharmacological therapies [1]. IPF is characterised by excessive fibroblast proliferation leading to the formation of fibroblast foci secreting extracellular matrix, with subsequent disruption of pulmonary structure and function. Despite progress in the understanding of the pathogenesis of IPF, novel treatment modalities show limited efficacy and the prognosis of IPF only slowly improves, with current 5 -year mortality rates still ranging from $70 \%$ to $80 \%$. To boost prognosis and to rise above incremental reductions in mortality rates of IPF patients, innovative and unorthodox treatment modalities are eagerly awaited.

Key hallmarks of IPF, like uncontrolled proliferation, disturbed cell-to-cell communication, constitutive activation of intracellular signal transduction pathways and resistance to apoptosis, are reminiscent of those observed in cancer. Based on the similarities in pathogenic mechanisms, IPF has been proposed to behave like a malignant disorder of the lung [2]. In line with this notion, IPF and cancer share several cellular and molecular aberrations, like epigenetic and genetic changes, delayed apoptosis, altered response to regulatory signals, deregulated microRNA expression, reduced cell-cell communication and activation of specific developmental or remodelling pathways [3]. Envisioning IPF as a cancer-like disease may indeed be an interesting and unorthodox approach in the fight against IPF as it would allow to take advantage of the enormous knowledge in cancer biology and to treat IPF patients with drugs known to effectively limit cancer progression [4]. Importantly however, several arguments against IPF as cancer-like disease have been put forward of which the lack of metastasis in IPF as opposed to cancer is most mentioned although it is important to realise that not all cancers metastasise. Consequently, the validity to consider IPF as a cancer-like disorder is under debate and no consensus has been reached yet. In the current study, we explored the similarity between IPF and cancer at the transcriptome level by comparing gene expression datasets of IPF and non-small cell lung cancer (NSCLC) (the most common type of lung cancer, accounting for $85-90 \%$ of all lung cancers) patients.

To derive genes differentially expressed between IPF patients and nondiseased controls, we selected the two largest IPF gene expression datasets available containing 119 patients and 50 controls (GSE32537 [5]) and 160 patients and 108 controls (GSE47460). Using the R2 microarray analysis and visualisation platform (http://r2.amc.nl), we identified 1251 genes that were differentially expressed in the GSE32537 set and 2064 genes that were differentially expressed in the GSE47460 set (with a statistical cut-off of false discovery rate-corrected $p$-values $<0.01$ and a fold change $>1.5$ ) (figure 1a). The subsequent comparison of the differentially expressed genes led to an IPF gene signature containing 771 genes that are consistently up- or downregulated in IPF patients as compared to controls. Analysis of this IPF gene signature in two of the largest NSCLC datasets available, containing expression data of 46 tumour and 45 nontumour samples (GSE18842 [6]) and 91 tumour and 65 nontumour samples (GSE19188 [7]), revealed that 512 of the 771 genes were also differently expressed in both NSCLC datasets. Interestingly, however, only 123

@ERSpublications

Despite promising examples of anticancer drugs as potential treatment modalities for IPF, these transcriptome data argue against the general nature of anticancer drugs as anti-IPF drugs http://ow.ly/HjsV30nbcji

Cite this article as: Spek CA, Duitman JW. Is idiopathic pulmonary fibrosis a cancer-like disease? Transcriptome analysis to fuel the debate. ERJ Open Res 2019; 5: 00157-2018 [https://doi.org/ 10.1183/23120541.00157-2018].

Copyright $\odot$ ERS 2019. This article is open access and distributed under the terms of the Creative Commons Attribution Non-Commercial Licence 4.0. 
a)

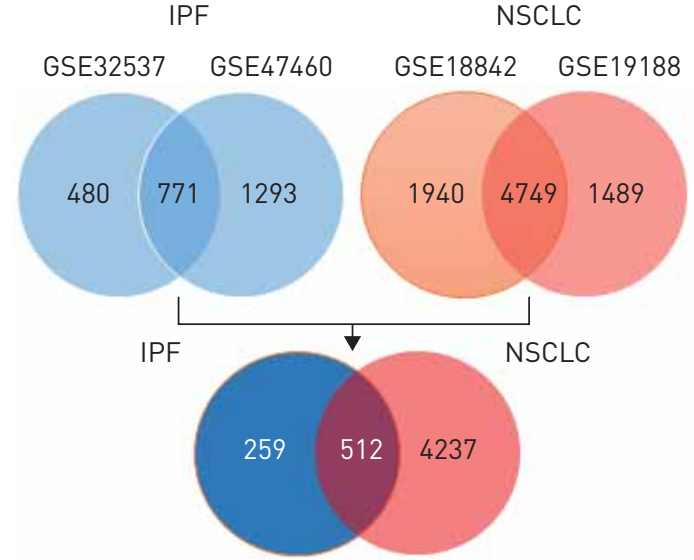

c)

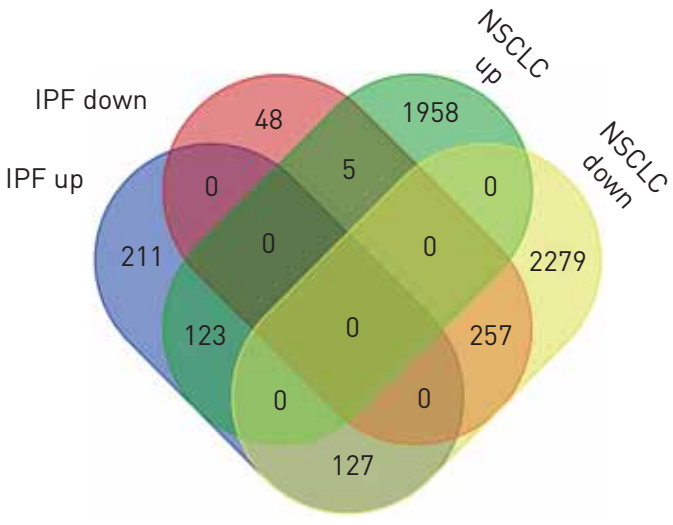

b)

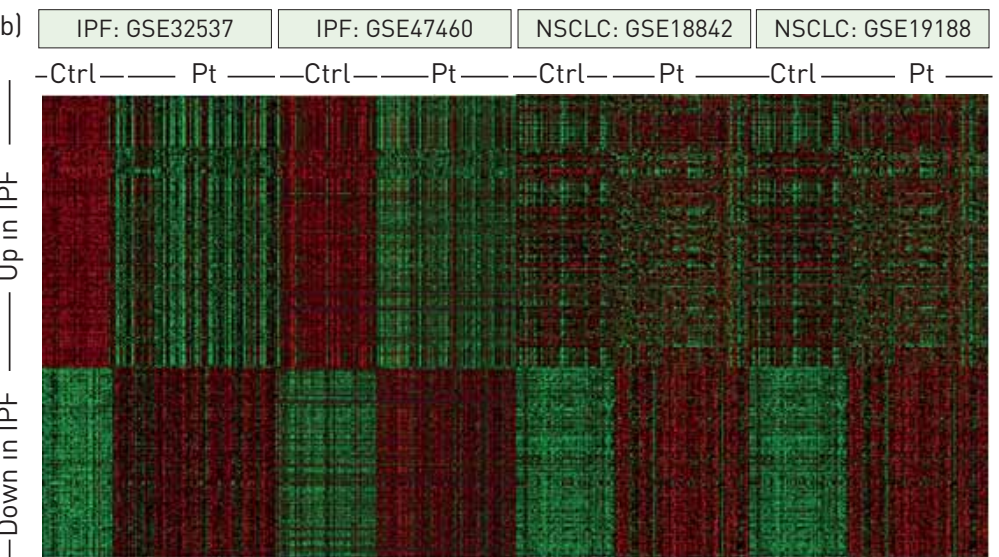

\begin{tabular}{|l|c|c|}
\hline \multicolumn{1}{|c|}{ Gene ontology category } & p-value & $\begin{array}{c}\text { FDR-corrected } \\
\text { p-value }\end{array}$ \\
\hline Up-up gene cluster (123 genes) & & \\
1) Collagen catabolism & $3.00 \times 10^{-10}$ & $4.50 \times 10^{-7}$ \\
2) Cell adhesion & $4.30 \times 10^{-8}$ & $6.60 \times 10^{-5}$ \\
3) Extracellular matrix organisation & $5.80 \times 10^{-7}$ & $8.90 \times 10^{-4}$ \\
\hline Up-down cluster (127 genes) & & \\
1) Cilium movement & $2.10 \times 10^{-7}$ & $2.80 \times 10^{-4}$ \\
2) Cilium organisation & $7.60 \times 10^{-7}$ & $1.00 \times 10^{-3}$ \\
\hline Down-down cluster (257 genes) & & \\
1) Angiogenesis & $6.60 \times 10^{-7}$ & $1.10 \times 10^{-3}$ \\
\hline
\end{tabular}

FIGURE 1 Comparison between idiopathic pulmonary fibrosis (IPF) and non-small cell lung cancer (NSCLC) transcriptomes. a) Venn diagrams showing the overlap in differently expressed genes (false discovery rate (FDR)-corrected p-values $<0.05$ and a fold change $>1.5$ ) in the GSE32537 and GSE47460 IPF datasets (upper left), the GSE18842 and GSE19188 NSCLC datasets (upper right) and the overlap in differentially expressed IPF and NSCLC genes (lower Venn diagram). b) Heatmap of the 771 genes differently regulated genes in IPF (455 upregulated and 316 downregulated) in the different IPF and NSCLC datasets. Green denotes low expression and red denotes high expression. c) Venn diagram of the 512 genes differentially expressed both in the IPF and NSCLC datasets showing that 123 genes were upregulated in both IPF and NSCLC, 257 genes were downregulated in both IPF and NSCLC, 127 genes were upregulated in IPF but downregulated in NSCLC and five genes were downregulated in IPF but upregulated in NSCLC. d) Gene ontology enrichment analysis of the different (i.e. upregulated-upregulated, upregulated-downregulated and downregulated-downregulated for IPF and NSCLC) gene signatures. Ctrl: control; Pt: patient.

genes were upregulated in both IPF and NSCLC whereas even a larger proportion of genes upregulated in IPF patients $(n=127)$ was actually downregulated in NSCLC patients (figure $1 \mathrm{~b}$ and $\mathrm{c})$. Of the genes downregulated in IPF, the vast majority was also downregulated in NSCLC and only five of these genes were upregulated in NSCLC. The IPF gene expression profile thus seems to partly overlap with NSCLC profiles but especially genes upregulated in both patient groups are relatively scarce and outnumbered by genes that are upregulated in IPF but downregulated in NSCLC.

To determine whether the different gene signatures are associated with specific biological processes, gene ontology enrichment analysis was performed [8]. As shown in figure 1d, the 123 gene signature of genes upregulated in both IPF and NSCLC that contains, amongst others, collagens and metalloproteases grouped into gene ontology categories like cell adhesion, extracellular matrix organisation and collagen catabolism (figure 1d). Although this may not be surprising as all these processes are well known to be altered in both IPF and cancer, it suggests that drugs targeting the extracellular matrix or stromal compartment may hold promise in both disease entities. The 257 genes downregulated in both IPF and NSCLC were only significantly enriched in the angiogenesis gene ontology category. Obviously, angiogenesis is a key determinant in NSCLC and the antiangiogenic monoclonal antibody bevacizumab is a well-established treatment option for NSCLC patients [9]. Notably, deregulated angiogenesis is also a key phenomenon in IPF but the actual importance remains elusive as angiogenesis may exert both pro- and antifibrotic responses [10]. The 127 gene signature of genes upregulated in IPF but downregulated in NSCLC patients grouped into gene ontology categories related to ciliogenesis (i.e. cilium movement and cilium organisation). This may be of particular interest as excessive ciliogenesis has been suggested to contribute to fibrogenesis during IPF [11], whereas loss of cilia has been associated with the onset of 
malignancy in some human tumours [12]. Alternative studies suggest, however, that cilia appear to be necessary for cancer progression and a recent study shows that ciliogenesis mediates drug resistance [13], highlighting that the role of cilia in cancer is not straightforward.

Overall, a picture seems to emerge that IPF and NSCLC are rather divergent on the global transcriptome level but a limited number of genes show similar regulation. As these genes, however, group in gene ontology categories well known to play key roles in both IPF and cancer, it could well be that specific treatments already successfully employed in cancer patients may hold promise in the setting of IPF as well. The Food and Drug Administration approval of nintedanib, a tyrosine kinase inhibitor traditionally used to treat cancer patients, in the setting of IPF is obviously the best example of the applicability of cancer drugs in IPF. Several other drugs developed as anticancer agents, like GSK2126458 (a potent inhibitor of phosphoinositol 3-kinase and mammalian target of rapamycin) and UCN-01 (a competitive ATP inhibitor targeting several kinases that also reactivates FoxO3), also show great promise in pre-clinical models of IPF $[14,15]$, suggesting that alternative anticancer drugs may ultimately be used in the treatment of IPF. Despite these promising examples of anticancer drugs as potential treatment modalities for IPF, we believe that the transcriptome data argue against the general nature of anticancer drugs as anti-IPF drugs. Cancer drugs targeting the 123 genes of IPF- and NSCLC-induced signature may be attractive candidates in the setting of IPF but drugs targeting genes of the other signatures do not seem first-choice treatments. Overall, designating IPF as a cancer-like disease is interesting and unorthodox but IPF specific treatments may hold greater promise. The main advantage of designating IPF as a cancer-like disease may therefore be an increased awareness of this deadly disease [4] leading to additional funds allowing the most talented researchers to work on IPF.

\section{Arnold Spek and JanWillem Duitman}

Amsterdam UMC, University of Amsterdam, Center for Experimental and Molecular Medicine, Amsterdam, The Netherlands.

Correspondence: C. Arnold Spek, Amsterdam UMC, University of Amsterdam, Center for Experimental and Molecular Medicine (H2-257), Meibergdreef 9, 1105 AZ Amsterdam, The Netherlands.

E-mail: c.a.spek@amc.uva.nl

Received: Sept 102018 | Accepted after revision: Nov 292018

Conflict of interest: C.A. Spek has nothing to disclose. J. Duitman reports grants from Netherlands Organisation for Scientific Research during the conduct of the study.

Support statement: This work was supported by the Netherlands Organisation for Scientific Research (VENI grant 016.186.046). Funding information for this article has been deposited with the Crossref Funder Registry.

\section{References}

$1 \quad$ King TE Jr, Pardo A, Selman M. Idiopathic pulmonary fibrosis. Lancet 2011; 378: 1949-1961.

2 Vancheri C, Failla M, Crimi N, et al. Idiopathic pulmonary fibrosis: a disease with similarities and links to cancer biology. Eur Respir J 2010; 35: 496-504.

3 Vancheri C. Common pathways in idiopathic pulmonary fibrosis and cancer. Eur Respir Rev 2013; 22: 265-272.

4 Vancheri C. Idiopathic pulmonary fibrosis and cancer: do they really look similar? BMC Med 2015; 13: 220.

5 Yang IV, Coldren CD, Leach SM, et al. Expression of cilium-associated genes defines novel molecular subtypes of idiopathic pulmonary fibrosis. Thorax 2013; 68: 1114-1121.

6 Sanchez-Palencia A, Gomez-Morales M, Gomez-Capilla JA, et al. Gene expression profiling reveals novel biomarkers in nonsmall cell lung cancer. Int J Cancer 2011; 129: 355-364.

7 Hou J, Aerts J, den Hamer B, et al. Gene expression-based classification of non-small cell lung carcinomas and survival prediction. PLoS One 2010; 5: e10312.

8 Huang DW, Sherman BT, Lempicki RA. Bioinformatics enrichment tools: paths toward the comprehensive functional analysis of large gene lists. Nucleic Acids Res 2009; 37: 1-13.

9 Russo AE, Priolo D, Antonelli G, et al. Bevacizumab in the treatment of NSCLC: patient selection and perspectives. Lung Cancer (Auckl) 2017; 8: 259-269.

10 Hanumegowda C, Farkas L, Kolb M. Angiogenesis in pulmonary fibrosis: too much or not enough? Chest 2012; 142: 200-207.

11 Lee J, Oh DH, Park KC, et al. Increased primary cilia in idiopathic pulmonary fibrosis. Mol Cells 2018; 41: 224-233.

12 Basten SG, Giles RH. Functional aspects of primary cilia in signaling, cell cycle and tumorigenesis. Cilia 2013; $2: 6$.

13 Jenks $\mathrm{AD}$, Vyse S, Wong JP, et al. Primary Cilia mediate diverse kinase inhibitor resistance mechanisms in cancer. Cell Rep 2018; 23: 3042-3055.

14 Mercer PF, Woodcock HV, Eley JD, et al. Exploration of a potent PI3 kinase/mTOR inhibitor as a novel anti-fibrotic agent in IPF. Thorax 2016; 71: 701-711.

15 Al-Tamari HM, Dabral S, Schmall A, et al. FoxO3 an important player in fibrogenesis and therapeutic target for idiopathic pulmonary fibrosis. EMBO Mol Med 2018; 10: 276-293. 